iron could only have condensed on the inside of the tube in which the metal was contained.

Oxygen, made by electrolysis of pure baryta solution, was finally allowed to enter the vessels. The paraffin wax used to line the quartz and glass vessels was purified by boiling with many changes of " conductivity" water, with frequent shaking, for about roo hours. The iron used was a commercial electrolytic sheet iron containing $99 \cdot 9$ per cent. iron. It was carefully cleaned with finely divided carborundum.

The results were the same in the quartz vessels and in the quartz and glass vessels lined with paraffin wax. Rusting was visible in all cases within a few hours.

It was only when pure iron, made as described in the paper, was used that no rusting took place.

After these and other experiments, it was considered that quartz vessels, which lend themselves admirably to cleaning and purification, were the most suitable vessels in which to study the reaction.

It is claimed by the authors that they have established the following facts:-(i) Pure iron does not rust when placed in contact with pure water and pure oxygen. (The term pure is used, of course, in a relative sense.) (2) The contention that "the dominant factor in the atmospheric corrosion of commercial iron is carbonic acid," or any other acid, is untrue. This must not be taken to mean, however, that carbonic acid plays no part in the atmospheric corrosion of ordinary commercial iron.

Bertram Lambert.

Chemical Department, University Museum, Oxford.

THE reference made to Mr. Lambert's work in my note on the rusting of iron has already proved of value in eliciting from the author a statement in reference to tests carried out in vessels lined with purified paraffin wax. His statement does not, however, provide a solution of the problem to which attention was directed, namely, that commercial iron exposed freely to air and water under the conditions described by Moody and by Friend does not rust, whereas in his own experiments rusting took place in the case of all but the most highly purified samples. It is difficult to predict whether the essential difference which still awaits discovery will ultimately be found in the iron, in the air, in the water, or in the vessel, but it is to be hoped that further discussion-in the columns of NATURE or elsewhere-may speedily throw light on this difficult and important matter.

It is a common experience, of which Mr. Lambert's work has provided excellent illustrations, that an unsuspected impurity (such as platinum in iron) is far more likely to promote than to prevent chemical change. It was for this reason that attention was directed first to the experiments in which rusting actually took place rather than to those in which it was successfully prevented. My suggestion in reference to silicic acid is correctly described, but I do not think that a note of exclamation was called for in view of the fact that mere contact with glass, as Moody found, actually produces the effects referred to, not in oxygen, but in purified air. The quantity of acid required to initiate the rusting must be extremely small, but its complete removal seems to outweigh in importance every other factor that may be taken into consideration. This view is amply supported by the fact that Friend, using common iron, common air, and the simplest apparatus, was able to stop the rusting by the simple device of washing the iron with a common alkali and then rinsing it with common water evaporated from the alkali and condensed on the iron.

With the first of Mr. Lambert's claims I am entirely in agreement; his second claim I regard as unproved and fallacious. To the statement that "the dominant factor in the atmospheric corrosion of commercial iron is carbonic acid" I adhere unreservedly, not only because the corrosion can be stopped by the addition of an alkali or by the removal of acid, but even more emphatically because the carbonic acid leaves behind an irrefutable proof of its activity in the presence of something like ro per cant. of ferrous carbonate in the rust formed under the commonplace conditions to which I referred.

T. M. L.

\section{April Meteors.}

The following are the most important meteoric events that become due from about April 6 to April 30 in the present year :-

Epoch April 5, $2 \mathrm{Ih}$. (G.M.T.), approximately second order of magnitude. Principal maximum April 6, 2 xh. $55 \mathrm{~m}$.; secondary maximum April 6, ih.

Epoch April 7, I7h, eighteenth order of magnitude. Principal maximum April 9, gh. 4om.; secondary maxima April 8, 2h. I5m., April 8, ixh. 30m., and April 9, 2 Ih. $30 \mathrm{~m}$.

Epoch April 1o, 17h., twenty-third order of magnitude. Principal maximum April 9, oh. I5m.; secondary maxima April 8, 21h. $15 \mathrm{~m}$. and April 10, 14h. 3om.

Epoch April II, 4h., twenty-eighth order of magnitude. Principal maximum April II, IIh. I5m.; secondary maximum April 12, 7h. $15 \mathrm{~m}$.

Epoch April 13, 22h. 3om., thirty-third order of magnitude. Principal maximum April 14, I5h. 40m.; secondary maximum April 15 , 14h. $40 \mathrm{~m}$.

Epoch April 17, 17h., twenty-ninth order of magnitude. Principal maximum April 18, 2h. 151.1.; secondary maximum April i8, 4h. 5om.

Epoch April 19, 8h., twenty-fourth order of magnitude. Principal maximum April 17, I3h. 40m.; secondary maximum April 17, $2 \mathrm{rh} .45 \mathrm{~m}$.

Lyrid epoch April I9, I4h. 30m., approximately eighth order of magnitude. Principal maxima April $\mathbf{1 7}$, 20h. 50m., and April 19, 9h. 50m.; secondary maxima April r9, 9h. 3om., and April 19, irh. 40m.

Epoch April 23, 3h. Iom., approximately sixth order of magnitude. Principal maximum April 21, x6h. 40m. secondary maxima April $2 \mathrm{I}, 3 \mathrm{~h}$. $55 \mathrm{~m}$., and April 22, 2oh. $30 \mathrm{~m}$.

Epoch April 25, 6h. 3om., eleventh order of magnitude. Principal maximum April 23, I3h.; secondary maxima April 23, 23h. 3om., and April 24, 22h. 5om.

Epoch April 26, 6h., thirty-first order of magnitude. Principal maximum April 27, 12h. Iom.; secondary maximum April 27, 4h. 40m.

Epoch April 27, th., approximately tenth order of magnitude. Principal maximum April 28, 22h. 5om.; secondary maximum April $27,23 \mathrm{~h}$. $50 \mathrm{~m}$.

Shooting stars should be numerous on April 19, as several maxima occur shortly before midnight on this date, among which Lyrids and bright meteors from allied radiants will probably be strongly in evidence. There is also another Lyrid display on the night of April 21, but it will only be partially visible on this side of the Atlantic.

April 3.

John R. Henry.

\section{Insurance against Rain.}

As this scheme of insurance is one of the few of which data can be obtained by "the man in the street". from which the odds in favour of the underwriters can be calculated, I went to the trouble of investigating the matter. Possibly the results may interest your readers.

In "British Rainfall" for 1909, by Dr. Hugh Robert Mill, statistics are given of the number of days on which varying quantities of rain fell. The figures give the average for thirty-seven rainfall stations in England and Wales over a period of seven years, 1903-9. From these, by a method of interpolation, it is found that fifty-eight days in a year may be expected to show a fall of 0.20 of an inch or more, and seventy-four of 0.15 or more.

Working out the probabilities on the basis of these figures, assuming that rain on one day is independent of rain on any other day, the following results are obtained :-for every $100 l$. received in premiums the underwriters may expect to have to pay, in the case of Policy A, 66l.; Policy B, 74l.; Policy C, $43 l$.; and Policy D, $64 l$.

In favour of the underwriters is the fact that in the south and south-east, where alone the scheme is applicable, the probability of rain is less than in England and Wales generally. A further point in their favour is that the summer months are less wet than the mean of the whole year on which the above figures are calculated. C. O. Bartrum. NO. 2 I62, VOL. 86]

32 Willoughby Road, Hampstead, N.W., March 30. 